\title{
What can We Expect for the Therapy of Neurological and Psychiatric Diseases in 2018?
}

\section{Leandro Bueno Bergantin*}

Laboratory of Autonomic and Cardiovascular Pharmacology, Department of Pharmacology, Escola Paulista de Medicina, Universidade Federal de São Paulo (UNIFESP), Rua Pedro de Toledo, São Paulo- Brazil

\section{Editorial}

This editorial brings the expectations, and my impressions, for the field of neurological and psychiatric diseases in 2018

Another year has begun, and the world still faces the challenge for treating neurological and psychiatric diseases. What can we expect for the field in this year? Indeed, I am now very busy in concluding my new book (second edition) about the "calcium paradox" discovery, including its impact in neurological and psychiatric diseases. While I am writing this editorial, this new book has been finalized. The contract has been signed, and everything has been running properly. What can we expect from this new book?

\section{News Coming Soon}

In fact, this new book brings novel insights for treating neurological and psychiatric diseases. It compiles more than 300 references in the field, including more than 30 references of my own authorship. This second edition has amplified the concept of the "calcium paradox" discovery, herein discussing other neurodegenerative diseases like Huntington's disease, Amyotrophic Lateral Sclerosis and Spinal muscular atrophy. What do these diseases have in common? Calcium ion is the answer! Now we know that calcium ion signalling is dysregulated in such diseases, and an excess of this ion within neurons is deleterious [1-4]. Why? Because calcium ion may activate, for example, caspases, thus achieving routes of death within the cells. So, by reducing the excess of calcium ion, we could indeed reduce neuronal death, and thus alleviating neurodegenerative diseases symptoms [14]. Virtually, many neurodegenerative diseases present an excess of calcium ions within neurons, thus this approach (reducing calcium ions) could be a "universal therapy" for these diseases. This new book discusses this issue! Indeed, it goes beyond, and discusses the role of the interaction between calcium ion and cAMP signalling pathways, another important second messenger. My entire career has been developed in this field, which indeed began with skeletal muscle, there by the year of 2008 [5]. Then, after developing a novel experimental model to study skeletal muscle protein degradation and confirming that elevating cAMP levels can reduce muscle wasting, I shifted to smooth muscle field, and later to peripheral nervous system, and nowadays to central nervous system [1-4]. In all these situations, calcium ion and cAMP second messengers followed me! And here I am, studying the role of calcium ion and cAMP signalling interaction in central nervous system, thus pursuing a new venue to treat neurological and psychiatric diseases: through the pharmacological modulation of the calcium ion and cAMP signalling pathways. The results that our group has been obtaining are promising, and indeed have been dramatically impacting the field! The great benefit of our approach is the possibility of using a drug already approved for hypertension (calcium channel blockers) into another usage, neurodegenerative diseases. This approach has been proving to work properly because calcium ions are accepted as universal messengers that control many functions, since contraction to neurotransmitter/hormones release. Then, when this ion becomes dysregulated within cells, its toxic effects arise, thus growing into diseases, like neurodegenerative diseases. Again, yes, reducing calcium ions within neuronal cells may be the answer for treating these diseases!

Finally, I hope you enjoy my new book, it has been conceived with all my passion coming from my heart! Indeed, neurological and psychiatric patients deserve it, then I also dedicate this book to them!

*Corresponding author: Leandro Bueno Bergantin, Laboratory of Autonomic and Cardiovascular Pharmacology, Department of Pharmacology, Escola Paulista de Medicina, Universidade Federal de São Paulo (UNIFESP), Rua Pedro de Toledo, 669-Vila Clementino, São Paulo-SP, Brazil, Tel: 5511 5576-4973; Email: leanbio39@yahoo.com.br

Received February 04, 2018; Accepted February 08, 2018; Published February 12,2018

Citation: Bergantin LB (2018) What can We Expect for the Therapy of Neurological and Psychiatric Diseases in 2018? Brain Disord Ther 7: e126. doi: 10.4172/2168-975X.1000e126

Copyright: $\odot 2018$ Bergantin LB. This is an open-access article distributed under the terms of the Creative Commons Attribution License, which permits unrestricted use, distribution, and reproduction in any medium, provided the original author and source are credited. 